\title{
Test of reliability of echocardiographic estimation of left ventricular dimensions and volumes
}

\author{
D. H. Bennett ${ }^{1}$ and D. J. Rowlands \\ From the University Department of Cardiology, The Royal Infirmary, Manchester
}

This test is based on the incompressibility of myocardium, which dictates that left ventricular wall volume remains constant throughout the cardiac cycle.

The volumes occupied by the left ventricular cavity, by ventricular wall plus cavity, and hence by ventricular wall alone were estimated, both at end-systole and at end-diastole, from echocardiographic measurements of cavity transverse dimension and wall thickness. Wall volumes were determined by assuming an ellipsoid shape (the major axis being predicted from regression equations relating angiocardiographic and echocardiographic cavity dimensions) and also by the cube method. A discrepancy between systolic and diastolic wall volume estimates indicates either that the measurements of ventricular dimensions were unreliable or that the assumptions of ventricular geometry involved in the volume calculations were incorrect.

Studies were made on 60 subjects. Using the ellipsoid formula, values for wall volume ranged from 66 to $719 \mathrm{ml}$; systolic and diastolic wall volumes correlated closely $(r=0.96$, mean difference $=6.8 \pm 0.9$ (SEM) \%) supporting the reliability of the echocardiographic dimensions and estimates of cavity and wall volume. In the 12 patients with very large end-diastolic cavity transverse dimensions $(6.5$ to $8.6 \mathrm{~cm})$ however, correlation was less good $(r=0 \cdot 81$, mean difference $=14 \cdot 3 \pm 2 \cdot 3$ (SEM) \%). Using the cube method, which does not allow for the changing relation between minor and major cavity axes with increasing cavity size, wall volumes were greater (76 to $986 \mathrm{ml}$ ) but correlation was similar $(r=0.94$, mean difference $=7 \cdot 1 \pm 0.9$ (SEM) \%).

Having established that it is possible to obtain close agreement between wall volumes determined at different points in the cardiac cycle, this test can be used to assess the reliability of echocardiographic left ventricular dimensions and volume estimates in individual subjects.

The noninvasive technique of echocardiography can be used to estimate several indices of left ventricular function including stroke volume, ejection fraction, ventricular mass, and wall stress. There are two main problems in the echocardiographic assessment of left ventricular function. The first problem is the difficulty which can be encountered in the recording and in the correct interpretation of left ventricular echocardiograms. The second problem lies in the fact that the estimation of left ventricular volumes is based on the measurement of a single cavity dimension and it, therefore, necessarily involves several assumptions as to the shape of the left ventricle. Though these assumptions are supported by angiocardiographic Received 3 June 1976.

'Present address: Cardiac Department, Radcliffe Infirmary, Oxford. observations (Greene et al., 1967; Sandler and Dodge, 1968; Gault, Ross, and Braunwald, 1968), the estimation of a three-dimensional shape from a single dimension is clearly open to criticism, particularly in patients with pronounced left ventricular cavity enlargement, where the cavity tends to be spherical rather than ellipsoid (Fortuin et al., 1971; Gibson, 1973), and in patients with an irregular or atypical cavity shape, as may occur in hypertrophic cardiomyopathy and ischaemic heart disease (Gibson, 1973; Feigenbaum, 1975).

We tested the reliability of the information which can be derived from the left ventricular echocardiogram, on a within-patient basis, by comparing diastolic and systolic estimates of left ventricular wall volume. The left ventricular wall, being non-gaseous, is incompressible and thus left ventricular wall volume must remain constant 
throughout the cardiac cycle. Wall volume can be estimated from echocardiographic measurements of wall thickness and cavity transverse dimension. Measurement of these two ventricular dimensions, and hence estimates of wall volume, can be made at end-diastole, when wall thickness is at a minimum and cavity size is at a maximum, and also at endsystole, when wall thickness is at a maximum and cavity size is at a minimum. If the ventricular dimensions are reliable, and if the assumptions of ventricular geometry involved in echocardiographic estimation of left ventricular volumes are correct, then diastolic and systolic estimates of wall volume should agree. Lack of agreement indicates either that the ventricular dimensions or geometric assumptions are unreliable, or that the echocardiogram is technically unsatisfactory or has been misinterpreted.

\section{Subjects and methods}

Left ventricular echocardiograms obtained from 8 normal subjects and from 52 patients with disorders likely to cause left ventricular enlargement were studied. The echocardiograms were selected for their clarity; in all records it was possible unequivocally to identify left ventricular endocardium and epicardium.

The echocardiograms were recorded by the standard method (Feigenbaum, 1972) on light sensitive paper (Kodak Linagraph, 1895), at a paper speed of $50 \mathrm{~mm} / \mathrm{s}$, using a Smith Kline $20 \mathrm{~A}$ ultrasonoscope, $2 \cdot 25 \mathrm{MHz} 10 \mathrm{~cm}$ focussed transducer, and a Honeywell 1856 strip chart recorder. End-diastolic (Dd) and end-systolic (Ds) cavity transverse dimension, and end-diastolic (Wd) and end-systolic (Ws) left ventricular wall thicknesses were measured with the aid of the simultaneously recorded calibration scale (Fig. 1). Cavity transverse dimension was measured as the distance between left ventricular septal and posterior wall endocardium. Wall thickness was measured as the distance between posterior wall endocardium and epicardium. End-diastole was defined as the time of onset of the QRS complex of the simultaneously recorded electrocardiogram, and end-systole as the point of maximum anterior movement of the posterior wall.

Left ventricular wall volumes were calculated, at end-diastole and end-systole, by subtracting the estimated cavity volume from the estimated volume occupied by ventricular wall plus cavity. Two different formulae were used.

\section{(1) Cube formula}

This formula is based on the empirical observation that the cube of the cavity transverse dimension $\left(D^{3}\right)$ approximates to the left ventricular cavity volume (Feigenbaum et al., 1969). By assuming uniform wall thickness (W), the volumes occupied by ventricular wall plus cavity $\left((2 \mathrm{~W}+\mathrm{D})^{3}\right)$ and hence by ventricular wall alone can be calculated, thus: wall volume $=(2 \mathrm{~W}+\mathrm{D})^{3}-\mathrm{D}^{3}$ (Bennett and Evans, 1974).

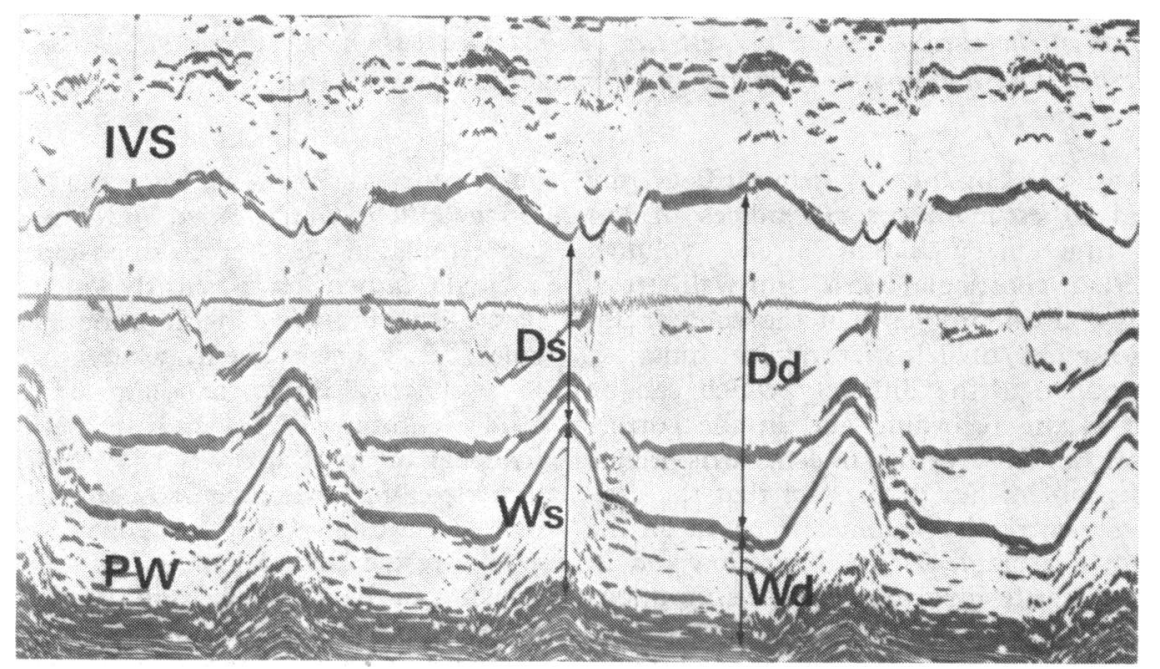

FIG. 1 Left ventricular echocardiogram (Case 26). IVS, interventricular septum; PW, posterior wall; Ws and Wd, end-systolic and end-diastolic wall thickness respectively; $D s$ and $D d$, end-systolic and end-diastolic cavity transverse dimension, respectively. 


\section{(2) Ellipsoid formula}

The empirical observation on the relation of the cube of the cavity transverse dimension to cavity volume can be explained by assuming (a) that the left ventricle has the shape of an ellipsoid of revolution, a widely held assumption in angiocardiographic work (Dodge et al., 1966; Greene et al., 1967; Sandler and Dodge, 1968), and (b) that the cavity transverse dimension is equal to each minor axis and to the semi-major axis (Chapelle and Mensch, 1969; Popp and Harrison, 1970; Troy, Pombo, and Rackley, 1972). If these relations hold, the minor radii are each $\mathrm{D} / 2$ and the major axis is $\mathrm{D}$, and the cavity volume becomes $4 / 3 \pi(\mathrm{D} / 2)^{2} \mathrm{D}$ $=1 \cdot 047 \mathrm{D}^{3}, \bumpeq \mathrm{D}^{3}$.

However, with cavity dilatation, the cavity minor axis increases relative to the major axis, the ventricle becoming more spherical in shape (Fortuin et al., 1971; Teichholz et al., 1972; Gibson, 1973). In addition, the relation between major and minor axes varies during the cardiac cycle (Gault et al., 1968). Gibson (1973), in a study of 50 patients with a variety of cardiac disorders, derived regres- sion equations relating angiocardiographic measurements of cavity major axis (L) to echocardiographic measurements of cavity transverse dimension (D), at end-diastole and end-systole:

$$
\mathrm{Ld}=5.90+0.98 \mathrm{Dd} ; \mathrm{Ls}=4 \cdot 18+1 \cdot 14 \mathrm{Ds} \text {. }
$$

These regression equations are similar to that derived from end-diastolic data by Ratshin, Rackley, and Russell (1974):

$$
\mathrm{Ld} / 2=2 \cdot 518+0 \cdot 462 \mathrm{Dd} \text {. }
$$

By predicting the cavity major axis from Gibson's regression equations, left ventricular wall volume can be estimated, in the same way as angiocardiographic measurements are made (Rackley et al., 1964), by using the formula for the volume of an ellipsoid. Thus: wall volume $=$

$$
4 / 3 \pi(\mathrm{W}+\mathrm{D} / 2)^{2}(\mathrm{~W}+\mathrm{L} / 2)-4 / 3 \pi(\mathrm{D} / 2)^{2} \mathrm{~L} / 2 \text {. }
$$

Correlation was sought between end-diastolic and end-systolic wall volume estimates, and the difference between these estimates was expressed as a percentage of the diastolic wall volume estimate. The subjects' clinical details, ventricular dimensions, and wall volume estimates are given in

\begin{tabular}{|c|c|c|c|c|c|c|c|c|}
\hline $\begin{array}{l}\text { Case } \\
\text { No. }\end{array}$ & Diagnosis & Age (y) & $s / d$ & $W(\mathrm{~cm})$ & $D(\mathrm{~cm})$ & $L(\mathrm{~cm})$ & $\begin{array}{l}\text { Wall v } \\
\text { Cube }\end{array}$ & $\begin{array}{l}\text { ee (ml) } \\
\text { Ellipsoid }\end{array}$ \\
\hline \multirow[t]{2}{*}{1} & Normal & 29 & s & 1.55 & $3 \cdot 30$ & $7 \cdot 95$ & 226 & 192 \\
\hline & & & d & 0.90 & $5 \cdot 35$ & $11 \cdot 15$ & 212 & 180 \\
\hline \multirow[t]{2}{*}{2} & Normal & 38 & s & $1 \cdot 35$ & $2 \cdot 80$ & $7 \cdot 50$ & 144 & 131 \\
\hline & & & d & 0.85 & $4 \cdot 45$ & $10 \cdot 25$ & 145 & 130 \\
\hline \multirow[t]{2}{*}{3} & Normal & 25 & $\mathbf{s}$ & 1.50 & $3 \cdot 10$ & $7 \cdot 70$ & 197 & 170 \\
\hline & & & $d$ & 0.95 & $4 \cdot 85$ & $10 \cdot 65$ & 194 & 168 \\
\hline \multirow[t]{2}{*}{4} & Normal & 27 & $\mathbf{s}$ & 1.55 & $2 \cdot 45$ & 6.95 & 156 & 140 \\
\hline & & & d & $1 \cdot 10$ & $3 \cdot 70$ & $9 \cdot 55$ & 155 & 146 \\
\hline \multirow[t]{2}{*}{5} & Normal & 25 & $\mathbf{s}$ & 1.55 & $3 \cdot 10$ & $7 \cdot 70$ & 213 & 186 \\
\hline & & & d & 0.95 & $4 \cdot 80$ & $10 \cdot 60$ & 190 & 166 \\
\hline \multirow[t]{2}{*}{6} & Normal & 20 & s & $1 \cdot 45$ & $2 \cdot 70$ & $7 \cdot 25$ & 156 & 139 \\
\hline & & & d & 0.95 & 3.90 & $9 \cdot 70$ & 136 & 127 \\
\hline \multirow[t]{2}{*}{7} & Normal & 18 & $\mathbf{s}$ & $1 \cdot 35$ & $3 \cdot 30$ & $7 \cdot 95$ & 180 & 155 \\
\hline & & & $\mathrm{d}$ & 0.95 & $4 \cdot 65$ & $10 \cdot 45$ & 181 & 159 \\
\hline \multirow[t]{2}{*}{8} & Normal & 18 & $\mathbf{s}$ & 1.50 & $3 \cdot 15$ & $7 \cdot 75$ & 201 & 173 \\
\hline & & & d & 0.95 & 5.00 & $10 \cdot 80$ & 204 & 175 \\
\hline \multirow[t]{2}{*}{9} & Coarct & 30 & $\mathbf{s}$ & 1.95 & $2 \cdot 75$ & $7 \cdot 30$ & 273 & 230 \\
\hline & & & d & $1 \cdot 25$ & $4 \cdot 60$ & $10 \cdot 40$ & 261 & 231 \\
\hline \multirow[t]{2}{*}{10} & Coarct & 31 & s & $2 \cdot 00$ & 3.05 & $7 \cdot 65$ & 322 & 266 \\
\hline & & & d & 1.05 & 6.00 & $11 \cdot 80$ & 315 & 255 \\
\hline \multirow[t]{2}{*}{11} & Coarct & 20 & $\mathbf{s}$ & $2 \cdot 40$ & 3.05 & $7 \cdot 65$ & 455 & 377 \\
\hline & & & d & 1.55 & $5 \cdot 35$ & $11 \cdot 15$ & 450 & 366 \\
\hline \multirow[t]{2}{*}{12} & Coarct & 17 & $\mathbf{s}$ & $2 \cdot 20$ & 3.00 & $7 \cdot 60$ & 378 & 308 \\
\hline & & & d & $1 \cdot 30$ & $5 \cdot 20$ & 11.00 & 334 & 278 \\
\hline \multirow[t]{2}{*}{13} & Coarct & 20 & $\mathbf{s}$ & $2 \cdot 05$ & $3 \cdot 15$ & $7 \cdot 75$ & 350 & 286 \\
\hline & & & $\mathrm{d}$ & $1 \cdot 40$ & $5 \cdot 15$ & 10.95 & 366 & 303 \\
\hline \multirow[t]{2}{*}{14} & Coarct & 17 & $\mathbf{s}$ & $2 \cdot 30$ & 3.00 & $7 \cdot 60$ & 412 & 333 \\
\hline & & & d & 1.45 & $5 \cdot 00$ & $10 \cdot 80$ & 368 & 306 \\
\hline \multirow[t]{2}{*}{15} & Coarct & 20 & $\mathbf{s}$ & $2 \cdot 40$ & 3.05 & $7 \cdot 65$ & 455 & 377 \\
\hline & & & d & 1.55 & $5 \cdot 35$ & $11 \cdot 15$ & 450 & 366 \\
\hline
\end{tabular}
Table 1.

TABLE 1 Diagnoses, systolic and diastolic ventricular dimensions, and wall volumes 
1136 Bennett and Rowlands

TABLE 1 continued

\begin{tabular}{|c|c|c|c|c|c|c|c|c|}
\hline \multirow{2}{*}{$\begin{array}{l}\text { Case } \\
\text { No. }\end{array}$} & \multirow[t]{2}{*}{ Diagnosis } & \multirow[t]{2}{*}{$\operatorname{Age}(y)$} & \multirow[t]{2}{*}{$s / d$} & \multirow[t]{2}{*}{$W(\mathrm{~cm})$} & \multirow[t]{2}{*}{$D(\mathrm{~cm})$} & \multirow[t]{2}{*}{$L(\mathrm{~cm})$} & \multicolumn{2}{|c|}{ Wall volume (ml) } \\
\hline & & & & & & & Cube & Ellipsoid \\
\hline 16 & Hypert & 53 & $\begin{array}{l}\mathrm{s} \\
\mathrm{d}\end{array}$ & $\begin{array}{l}2.50 \\
1.70\end{array}$ & $\begin{array}{l}2 \cdot 70 \\
4.90\end{array}$ & $\begin{array}{r}7 \cdot 30 \\
10 \cdot 70\end{array}$ & $\begin{array}{l}437 \\
454\end{array}$ & $\begin{array}{l}416 \\
374\end{array}$ \\
\hline \multirow[t]{2}{*}{17} & Hypert & 40 & $\mathbf{s}$ & $2 \cdot 60$ & 3.00 & $7 \cdot 60$ & 524 & 415 \\
\hline & & & d & 1.90 & $5 \cdot 00$ & $10 \cdot 80$ & 557 & 451 \\
\hline \multirow[t]{2}{*}{18} & Hypert & 30 & $\mathbf{s}$ & $2 \cdot 05$ & 3.00 & $7 \cdot 60$ & 331 & 273 \\
\hline & & & d & 1.45 & $4 \cdot 75$ & $10 \cdot 55$ & 341 & 288 \\
\hline \multirow[t]{2}{*}{19} & AS & 43 & s & 1.90 & 1.85 & $6 \cdot 30$ & 174 & 157 \\
\hline & & & d & $1 \cdot 20$ & 3.65 & $9 \cdot 50$ & 173 & 162 \\
\hline \multirow[t]{2}{*}{20} & AS & 33 & $\mathbf{s}$ & $2 \cdot 10$ & $2 \cdot 00$ & $6 \cdot 50$ & 230 & 202 \\
\hline & & & d & $1 \cdot 30$ & $3 \cdot 70$ & 9.50 & 199 & 183 \\
\hline \multirow[t]{2}{*}{21} & AS & 30 & s & $1 \cdot 70$ & $2 \cdot 70$ & $7 \cdot 25$ & 207 & 187 \\
\hline & & & d & $1 \cdot 10$ & $4 \cdot 10$ & $9 \cdot 90$ & 181 & 152 \\
\hline \multirow[t]{2}{*}{22} & AS & 19 & s & $1 \cdot 70$ & $3 \cdot 15$ & $7 \cdot 75$ & 250 & 210 \\
\hline & & & d & $1 \cdot 10$ & $5 \cdot 00$ & $10 \cdot 80$ & 248 & 212 \\
\hline 23 & AS & 44 & s & $2 \cdot 00$ & $2 \cdot 90$ & $7 \cdot 50$ & 304 & 254 \\
\hline & & & d & 1.50 & $4 \cdot 10$ & $9 \cdot 90$ & 289 & 253 \\
\hline 24 & AS & 52 & s & 2.00 & $2 \cdot 20$ & $6 \cdot 70$ & 228 & 198 \\
\hline & & & d & $1 \cdot 30$ & 4.00 & $9 \cdot 80$ & 223 & 201 \\
\hline 25 & AS & 58 & s & $2 \cdot 30$ & $2 \cdot 35$ & 6.85 & 323 & 270 \\
\hline & & & d & 1.60 & $4 \cdot 25$ & 10.05 & 337 & 290 \\
\hline 26 & AS & 18 & $\mathbf{s}$ & $2 \cdot 05$ & $2 \cdot 15$ & 6.65 & 234 & 205 \\
\hline & & & d & 1.35 & 4.05 & $9 \cdot 85$ & 241 & 215 \\
\hline 27 & AS & 19 & s & 1.75 & 1.85 & $6 \cdot 30$ & 147 & 134 \\
\hline & & & d & $1 \cdot 20$ & $3 \cdot 35$ & $9 \cdot 15$ & 153 & 147 \\
\hline 28 & AS & 43 & s & 1.90 & 1.85 & $6 \cdot 30$ & 174 & 157 \\
\hline & & & d & $1 \cdot 20$ & 3.65 & $9 \cdot 50$ & 173 & 162 \\
\hline 29 & AS, LVF & 58 & s & 1.90 & $7 \cdot 20$ & $12 \cdot 40$ & 958 & 690 \\
\hline & & & d & 1.35 & $8 \cdot 30$ & 14.05 & 759 & 554 \\
\hline 30 & AS, AR & 45 & s & $2 \cdot 20$ & $2 \cdot 60$ & $7 \cdot 10$ & 325 & 270 \\
\hline & & & d & 1.40 & $4 \cdot 65$ & $10 \cdot 45$ & 313 & 269 \\
\hline 31 & AS, AR & 47 & s & $2 \cdot 60$ & $3 \cdot 10$ & $7 \cdot 70$ & 542 & 427 \\
\hline & & & d & $1 \cdot 70$ & $5 \cdot 60$ & $11 \cdot 40$ & 553 & 440 \\
\hline 32 & AS, AR & 50 & s & 3.00 & 3.00 & $7 \cdot 60$ & 710 & 541 \\
\hline & & & d & $2 \cdot 30$ & $4 \cdot 80$ & $10 \cdot 60$ & 720 & 575 \\
\hline 33 & AS, AR & 52 & s & 1.55 & 4.50 & $9 \cdot 30$ & 348 & 276 \\
\hline & & & d & 1.25 & $5 \cdot 70$ & $11 \cdot 50$ & 366 & 297 \\
\hline 34 & AS, AR & 30 & $\mathbf{s}$ & $2 \cdot 80$ & 3.60 & $8 \cdot 30$ & 736 & 560 \\
\hline & & & d & $1 \cdot 80$ & $5 \cdot 90$ & $11 \cdot 70$ & 652 & 510 \\
\hline 35 & AS, AR & 36 & 8 & $2 \cdot 15$ & $2 \cdot 65$ & $7 \cdot 20$ & 317 & 264 \\
\hline & & & d & 1.50 & $5 \cdot 00$ & $10 \cdot 80$ & 387 & 321 \\
\hline 36 & AS, AR & 19 & s & 1.80 & $3 \cdot 50$ & $8 \cdot 15$ & 315 & 258 \\
\hline & & & d & $1 \cdot 30$ & $5 \cdot 00$ & $10 \cdot 80$ & 314 & 264 \\
\hline 37 & AS, AR, MS & 57 & s & $2 \cdot 15$ & $2 \cdot 45$ & 6.95 & 293 & 247 \\
\hline & & & d & 1.50 & $4 \cdot 25$ & 10.05 & 304 & 264 \\
\hline 38 & AS, AR, MS & 46 & s & $1 \cdot 80$ & 2.05 & $6 \cdot 50$ & 172 & 151 \\
\hline & & & d & 1.05 & 4.05 & $9 \cdot 85$ & 166 & 152 \\
\hline 39 & CAF & 26 & $\begin{array}{l}\mathbf{s} \\
\mathrm{d}\end{array}$ & 1.85 & 3.65 & $\begin{array}{r}8.35 \\
11.45\end{array}$ & 348 & 270 \\
\hline 40 & $\mathbf{A R}$ & 27 & $\mathrm{~s}$ & $\begin{array}{l}1.15 \\
1.90\end{array}$ & $\begin{array}{l}5.65 \\
3.45\end{array}$ & $\begin{array}{r}11 \cdot 45 \\
8 \cdot 10\end{array}$ & $\begin{array}{l}322 \\
340\end{array}$ & $\begin{array}{l}264 \\
277\end{array}$ \\
\hline 40 & AR & 21 & $\mathrm{~d}$ & $\begin{array}{l}1.90 \\
1.10\end{array}$ & $\begin{array}{l}3.45 \\
6 \cdot 10\end{array}$ & $\begin{array}{r}8 \cdot 10 \\
11.90\end{array}$ & 345 & 277 \\
\hline 41 & AR & 19 & s & 2.05 & 4.95 & $9 \cdot 80$ & 620 & 470 \\
\hline & & & $d$ & 1.35 & $7 \cdot 65$ & $13 \cdot 40$ & 661 & 492 \\
\hline 42 & AR & 46 & s & $2 \cdot 25$ & 4.90 & $9 \cdot 75$ & 713 & 537 \\
\hline & & & $\mathrm{d}$ & $1 \cdot 40$ & $7 \cdot 50$ & $13 \cdot 25$ & 671 & 501 \\
\hline 43 & AR & 37 & s & 1.60 & $3 \cdot 60$ & $8 \cdot 30$ & 268 & 222 \\
\hline & & & d & 1.00 & $5 \cdot 60$ & $11 \cdot 40$ & 263 & 216 \\
\hline 44 & AR & 6 & s & 1.00 & $2 \cdot 50$ & $7 \cdot 05$ & 76 & 66 \\
\hline & & & d & 0.65 & $3 \cdot 50$ & $9 \cdot 35$ & 68 & 69 \\
\hline 45 & AR & 32 & s & $2 \cdot 15$ & $3 \cdot 70$ & $8 \cdot 40$ & 461 & 365 \\
\hline 46 & & & d & $\begin{array}{l}1.05 \\
1.85\end{array}$ & $6 \cdot 80$ & $12 \cdot 60$ & 391 & 305 \\
\hline & AR & 56 & $\begin{array}{l}\mathrm{s} \\
\mathrm{d}\end{array}$ & $\begin{array}{l}1.85 \\
1.55\end{array}$ & $\begin{array}{l}5 \cdot 90 \\
7 \cdot 35\end{array}$ & $\begin{array}{l}10 \cdot 90 \\
13 \cdot 10\end{array}$ & $\begin{array}{l}679 \\
744\end{array}$ & $\begin{array}{l}506 \\
556\end{array}$ \\
\hline
\end{tabular}

Continued 
TABLE 1 continued

\begin{tabular}{|c|c|c|c|c|c|c|c|c|}
\hline $\begin{array}{l}\text { Case } \\
\text { No. }\end{array}$ & Diagnosis & Age (y) & $s / d$ & $W(\mathrm{~cm})$ & $D(\mathrm{~cm})$ & $L(\mathrm{~cm})$ & $\begin{array}{l}\text { Wall थ } \\
\text { Cube }\end{array}$ & $\begin{array}{l}\text { el (ml) } \\
\text { Ellipsoid }\end{array}$ \\
\hline \multirow[t]{2}{*}{47} & $\mathrm{AR}, \mathrm{MR}$ & 19 & s & 1.90 & $4 \cdot 70$ & 9.55 & 510 & 395 \\
\hline & & & d & $1 \cdot 30$ & $6 \cdot 60$ & $12 \cdot 35$ & 491 & 381 \\
\hline \multirow[t]{2}{*}{48} & AR, VSD & 23 & $\mathbf{s}$ & $2 \cdot 35$ & $5 \cdot 90$ & 10.90 & 986 & 719 \\
\hline & & & $d$ & 1.45 & $8 \cdot 55$ & $14 \cdot 30$ & 876 & 633 \\
\hline \multirow[t]{2}{*}{49} & AR, VSD & 17 & $\mathrm{~s}$ & $2 \cdot 00$ & $4 \cdot 20$ & $9 \cdot 00$ & 477 & 375 \\
\hline & & & d & $1 \cdot 10$ & 6.50 & $12 \cdot 50$ & 384 & 306 \\
\hline \multirow[t]{2}{*}{50} & AR, MS & 43 & $\mathbf{s}$ & 1.75 & $4 \cdot 55$ & $9 \cdot 35$ & 428 & 335 \\
\hline & & & d & 1.25 & $6 \cdot 40$ & $12 \cdot 15$ & 443 & 347 \\
\hline \multirow[t]{2}{*}{51} & AVR & 50 & $\mathbf{s}$ & 1.90 & $4 \cdot 30$ & $9 \cdot 10$ & 452 & 355 \\
\hline & & & d & $1 \cdot 10$ & $6 \cdot 80$ & $12 \cdot 60$ & 415 & 323 \\
\hline \multirow[t]{2}{*}{52} & AVR & 32 & $\mathbf{s}$ & 2.00 & $2 \cdot 35$ & 6.85 & 243 & 209 \\
\hline & & & $d$ & $1 \cdot 30$ & $4 \cdot 30$ & $10 \cdot 10$ & 249 & 219 \\
\hline \multirow[t]{2}{*}{53} & MR & 23 & s & $2 \cdot 00$ & $5 \cdot 00$ & $9 \cdot 90$ & 604 & 460 \\
\hline & & & d & 1.05 & $7 \cdot 80$ & 13.55 & 496 & 372 \\
\hline \multirow[t]{2}{*}{54} & MR & 52 & $s$ & 2.00 & $5 \cdot 25$ & $10 \cdot 15$ & 647 & 487 \\
\hline & & & d & 1.55 & $8 \cdot 10$ & $13 \cdot 85$ & 874 & 638 \\
\hline \multirow[t]{2}{*}{55} & MR & 51 & s & 1.95 & 3.45 & $8 \cdot 10$ & 356 & 289 \\
\hline & & & d & 1.20 & $5 \cdot 70$ & $11 \cdot 50$ & 346 & 282 \\
\hline \multirow[t]{2}{*}{56} & MR & 30 & $\mathbf{s}$ & 1.95 & 3.00 & $7 \cdot 60$ & 302 & 251 \\
\hline & & & $d$ & $1 \cdot 10$ & $5 \cdot 65$ & $11 \cdot 45$ & 303 & 249 \\
\hline \multirow[t]{2}{*}{57} & $M R, M S$ & 51 & $\mathbf{s}$ & 1.55 & $3 \cdot 70$ & $8 \cdot 40$ & 264 & 218 \\
\hline & & & d & 1.00 & $5 \cdot 55$ & $11 \cdot 35$ & 259 & 215 \\
\hline \multirow[t]{2}{*}{58} & PDA & 10 & s & 1.05 & 3.00 & $7 \cdot 60$ & 106 & 96 \\
\hline & & & d & 0.60 & $4 \cdot 70$ & $10 \cdot 50$ & 102 & 92 \\
\hline \multirow[t]{2}{*}{59} & PDA & 16 & s & 1.45 & $4 \cdot 25$ & 9.05 & 289 & 234 \\
\hline & & & d & 0.95 & $5 \cdot 70$ & $11 \cdot 50$ & 254 & 210 \\
\hline \multirow[t]{2}{*}{60} & COCM & 42 & $\mathbf{s}$ & 1.55 & $6 \cdot 15$ & $11 \cdot 20$ & 559 & 569 \\
\hline & & & d & 1.00 & $7 \cdot 50$ & $13 \cdot 25$ & 435 & 522 \\
\hline
\end{tabular}

$\mathrm{s}=$ end-systolic data; $\mathrm{d}=$ end-diastolic data; $\mathrm{W}=$ wall thickness; $\mathrm{D}=$ cavity transverse dimension; $\mathrm{L}=$ estimated maior axis; Coarct=aortic coarctation; hypert $=$ essential hypertension; $A S=$ aortic stenosis; $A R=$ aortic regurgitation; $M S=$ mitral stenosis; $M R=$ mitral regurgitation; $C A F=$ coronary artery fistula; $V S D=$ ventricular septal defect; $A V R==$ leakmg aortic prosthesis; $\mathrm{PDA}=$ persistent ductus arteriosus; $\mathrm{COCM}=$ congestive cardiomyopathy.

\section{Results}

Overall, there was close correlation between diastolic and systolic wall volume estimates using both the cube formula and the ellipsoid formula (Table 2). In some of the patients however, mainly those with pronounced cavity enlargement, agreement was poor. An example is shown in Fig. 2.

In subjects with normal or moderately enlarged cavities, wall volumes determined using the cube formula were slightly greater than those determined using the ellipsoid formula. In patients with very large cavities, where the predicted major axis was substantially less than twice the minor axis, wall volumes calculated using the cube formula were much greater than those determined from the ellipsoid formula.

\section{Discussion}

In most cases there was close agreement between estimates of left ventricular wall volume at endsystole, when wall thickness is at a maximum and cavity size is at a minimum, and at end-diastole, when wall thickness is at a minimum and cavity size is at a maximum. This supports the reliability of the wall thickness and cavity dimension measurements, and of the assumptions involved in the volume determinations. The agreement does not establish the reliability of the measurements, but it is certainly consistent with it, and lack of agreement is certainly inconsistent with reliability. In some cases, mainly those with very large ventricular cavities, agreement was poor indicating in these cases, either that the measurements of ventricular dimensions were unreliable or that the assumptions of ventricular geometry involved in the volume calculations could not be applied.

Though agreement was not improved by use of the method based on the ellipsoid formula, we feel that this method is likely to yield more realistic estimates of wall volume since it does take into account the changing relation between major and minor axes which occurs during the cardiac cycle and with increasing cavity size, whereas the cube method is based on a fixed relation between the two axes.

The test is based on the constancy of ventricular wall volume throughout the cardiac cycle. It might 
TABLE 2 Comparison of systolic and diastolic wall volume estimates

\begin{tabular}{lllllc}
\hline & $C / E$ & $n$ & $r$ & $P<$ & Mean difference (\%) \\
\hline All subjects & C & 60 & 0.94 & 0.001 & $7.1 \pm 0.9$ (SEM) \\
& E & & 0.96 & 0.001 & $6.8 \pm 0.9$ (SEM) \\
Subjects with C & 12 & 0.82 & 0.001 & $15.9 \pm 2.6$ (SEM) \\
Dd>6.5 cm & E & & 0.81 & 0.001 & $14.3 \pm 2.3($ SEM) \\
Subjects with C & 48 & 0.99 & 0.001 & $4.8 \pm 0.7($ SEM) \\
Dd $<6.5$ cm & E & & 0.98 & 0.001 & $4.9 \pm 0.7$ (SEM) \\
\hline
\end{tabular}

$\mathrm{C}$ and $\mathrm{E}=$ wall volumes estimated by cube and ellipsoid formulae respectively; $\mathrm{Dd}=$ end-diastolic cavity transverse dimension; $\mathbf{n}=$ number of subiects; $\mathbf{r}=$ correlation coefficient $\mathbf{P}=$ significance level; $\mathbf{S E M}=$ standard error of mean.

be argued that a change in wall volume could occur as a result of expulsion of blood from the myocardium during systole. Assuming a myocardial blood flow of $66 \mathrm{ml} / \mathrm{min}$ per $100 \mathrm{~g}$ (Brachfeld, Bozer, and Gorlin, 1959), i.e. approximately $1 \mathrm{ml} / \mathrm{s}$ per $100 \mathrm{~g}$, and using a mean transit time for the coronary circulation of 7 seconds (Gorlin, 1960), the coronary blood volume is calculated to be $7 \cdot 0 \mathrm{ml} / 100 \mathrm{~g}$. If the myocardium were completely emptied of blood during systole, it would only account for a 7 per cent change in wall volume. It is extremely unlikely that the myocardium is completely emptied of blood in systole. An assessment of the volume of blood likely to be expelled from the myocardium in systole can be obtained by a consideration of the total coronary flow. Using the above estimate for myocardial blood flow and assuming a heart rate of $60 /$ minute, only approximately $1 \mathrm{ml} \mathrm{blood} / \mathrm{min}$ per $100 \mathrm{~g}$ of myocardium can be expelled. Even if all this flow occurred between end-diastole and end-systole, it could only constitute $1 \mathrm{ml}$ blood volume to $100 \mathrm{~g}$ myocardial tissue or approximately 1 per cent. In view of the range of heart rates possible and the uncertain apportioning of blood between the left and right ventricular myocardium, it seems best to assume simply that any myocardial volume change related to expulsion of blood will account for between 1 and 7 per cent and will probably be much closer to the former than the latter.

Comparison of systolic and diastolic wall volume estimates has been used in angiocardiographic studies to assess the reliability of wall thickness and cavity volume measurements (Hugenholtz, Kaplan, and Hull, 1969; Lewis and Sandler, 1971). A difference of up to 25 per cent is usually regarded as acceptable (Lewis and Sandler, 1971; Sandler and Alderman, 1974). Agreement between wall volume estimates in this echocardiographic study was closer probably because of the tendency to

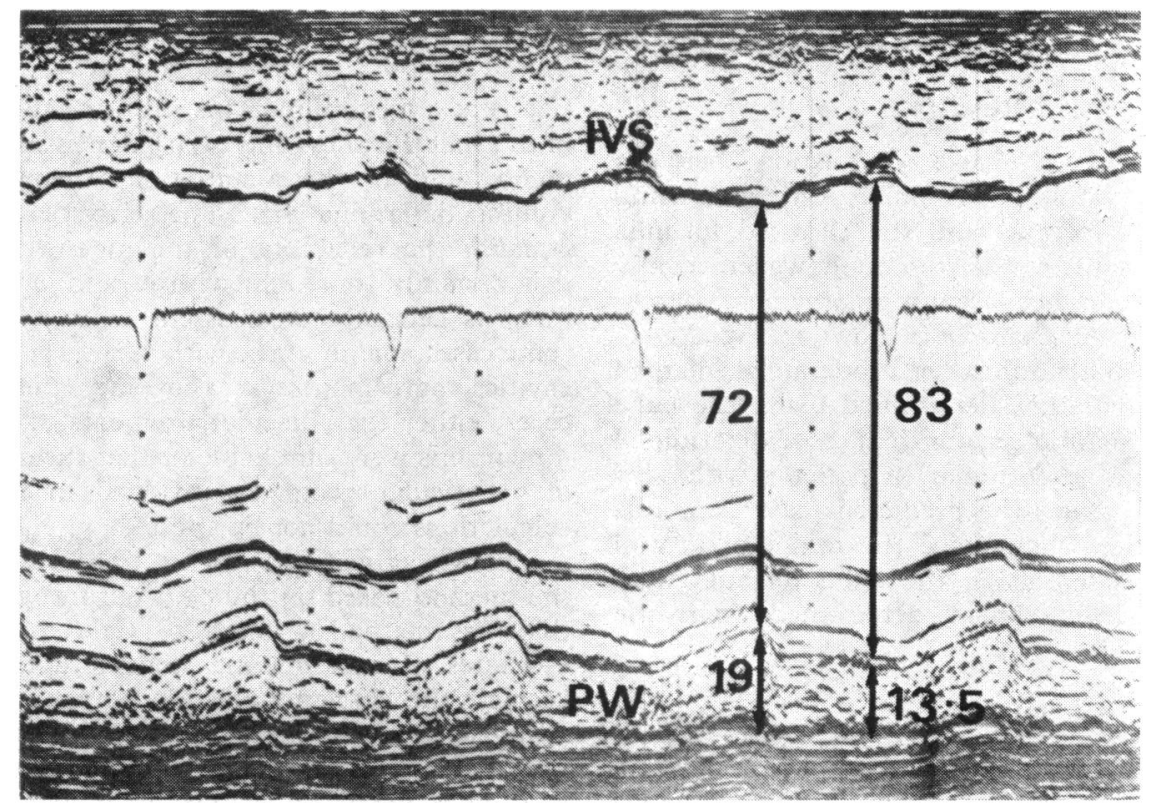

FIG. 2 Patient with severe left ventricular failure (Case 29). Distinct difference between systolic and diastolic estimates of wall volume using both cube $(26 \%)$ and ellipsoid $(25 \%)$ formulae. 
overestimate end-systolic wall thickness, and hence wall volume, by angiocardiography (Hugenholtz et al., 1969).

In conclusion, close agreement can be obtained between echocardiographic systolic and diastolic wall volume estimates. Having established this, comparison of wall volume estimates can be used to assess the reliability of measurements of ventricular dimensions and volumes in individual subjects. It is suggested that differences in excess of 10 per cent between systolic and diastolic left ventricular wall volume estimates point to lack of reliability of measurements of left ventricular volumes.

\section{References}

Bennett, D. H., and Evans, D. W. (1974). Correlation of left ventricular mass determined by echocardiography with vectorcardiographic and electrocardiographic voltage measurements. British Heart fournal, 36, 981.

Brachfeld, N., Bozer, J., and Gorlin, R. (1959). Action of nitroglycerin on the coronary circulation in normal and in mild cardiac subjects. Circulation, 19, 697.

Chapelle, M., and Mensch, B. (1969). Etude des variations du diamètre ventriculaire gauche chez l'homme par échocardiographie transthoracique. Archives des Maladies $d u$ Coeur et des Vaisseaux, 62, 1505.

Dodge, H. T., Sandler, H., Baxley, W. A., and Hawley, R. R. (1966). Usefulness and limitations of radiographic methods for determining left ventricular volume. American fournal of Cardiology, 18, 10.

Feigenbaum, H. (1972). Clinical applications of echocardiography. Progress in Cardiovascular Diseases, 14, 531.

Feigenbaum, H. (1975). Echocardiographic examination of the left ventricle. Circulation, 51, 1.

Feigenbaum, H., Wolfe, S. B., Popp, R. L., Haine, C. L., and Dodge, H. T. (1969). Correlation of ultrasound with angiocardiography in measuring left ventricular diastolic volume (abstract). American fournal of Cardiology, 23, 111.

Fortuin, N. J., Hood, W. P., Jr., Sherman, M. E., and Craige, E. (1971). Determination of left ventricular volumes by ultrasound. Circulation, 44, 575.
Gault, J. H., Ross, J., Jr., and Braunwald, E. (1968). Contractile state of the left ventricle in man. Instantaneous tension-velocity-length relations in patients with and without disease of the left ventricular myocardium. Circulation Research, 22, 451.

Gibson, D. G. (1973). Estimation of left ventricular size by echocardiography. British Heart fournal, 35, 128.

Gorlin, R. (1960). Measurement of coronary flow in health and disease. In Modern Trends in Cardiology, p. 191. Ed. by A. Morgan Jones. Butterworths, London.

Greene, D. G., Carlisle, R., Grant, C., and Bunnell, I. L. (1967). Estimation of left ventricular volume by one-plane cineangiography. Circulation, 35, 61 .

Hugenholtz, P. G., Kaplan, E., and Hull, E. (1969). Determination of left ventricular wall thickness by angiocardiography. American Heart fournal, 78, 513.

Lewis, R. P., and Sandler, H. (1971). Relationship between changes in left ventricular dimensions and the ejection fraction in man. Circulation, 44, 548.

Popp, R. L., and Harrison, D. C. (1970). Ultrasonic cardiac echography for determining stroke volume and valvular regurgitation. Circulation, 41, 493.

Rackley, C. E., Dodge, H. T., Coble, Y. D., Jr., and Hay, R. E. (1964). A method for determining left ventricular mass in man. Circulation, 29, 666.

Ratshin, R. A., Rackley, C. E., and Russell, R. O., Jr. (1974). Determination of left ventricular preload and afterload by quantitative echocardiography in man: calibration of the method. Circulation Research, 34, 711.

Sandler, H., and Alderman, E. (1974). Determination of left ventricular size and shape. Circulation Research, 34, 1.

Sandler, H., and Dodge, H. T. (1968). The use of single plane angiocardiograms for the calculation of left ventricular volume in man. American Heart fournal, 75, 325.

Teichholz, L. E., Kreulen, T. H., Herman, M. V., and Gorlin, R. (1972). Problems in echographic volume determinations: echo-angiographic correlations (abstract). Circulation, 46, Suppl. II, 75.

Troy, B. L., Pombo, J., and Rackley, C. E. (1972). Measurement of left ventricular wall thickness and mass by echocardiography. Circulation, 45, 602.

Requests for reprints to Dr. David Bennett, Cardiac Department, Radcliffe Infirmary, Oxford. 